\title{
Remote Innovation - Co-Creation During Times of Pandemic
}

Oliver Gerstheimer, Philipp Schütz, Philipp Englisch, Erhard Wimmer

Die COVID-19-Pandemie forderte 2020 die Wirtschaftswelt heraus und provozierte in vielen Unternehmen die Dezentralisierung und Digitalisierung der Denkarbeit. Co-Creation-Workshops mussten wirkungsvoll und schnell in Online-Formate übertragen werden. Für die Produktentwicklung und -innovation der Sartorius AG bedeutete dies die Entwicklung einer digitalen Plattform: Bestehende Inhalte und Prozesse wurden überarbeitet, verbessert, erweitert und in einer digitalen Infrastruktur zusammengeführt. Ausgehend von der Plattform erhalten Mitarbeiter:innen Zugang zu einem Methodenpool, Workshop-Vorlagen und Schulungsmaterialien. So können Teams weltweit produktiv zusammenarbeiten. Die digitale Plattform ist ganzheitlich und nachhaltig konzipiert und wird durch ein analoges Method Playbook und agile Schulungen, sowie eine Webcast-Serie ergänzt, in der weiterführende Informationen on-demand abgerufen werden können. Mit der Plattform wird die unternehmensweite Innovationskultur in Zeiten der Pandemie nicht nur erhalten, sondern sogar proaktiv ausgebaut und zusätzlich werden Zeit und Ressourcen gespart.

Keywords: Homeoffice, Remote Work, Co-Creation, Design Thinking, Innovationskultur, Pandemie

\section{Simplifying Progress - a digital challenge}

Kontinuierliche Innovation ist der Grundsatz von Sartorius. Dabei baut das Unternehmen auf Co-Creation und schöpft aus dem Design-Thinking-Pool. Das zentrale Leitprinzip Simplifying Progress gilt dabei nicht nur für die Kundenlösungen, sondern wird auch in der Produktentwicklung gelebt. Durch die gezielte Etablierung einer Innovation Culture arbeiteten interdisziplinäre Teams vor allem zeitlich und örtlich konzentriert, im Rahmen von Workshops an den unterschiedlichen Firmenstandorten zusammen.

Der Ausbruch der COVID-19-Pandemie im Jahr 2020 traf die Wirtschaft in großen Teilen unvorbereitet und bedeutete einen Einschnitt in die Innovationsstrategie vieler ambitionierter Unternehmen. Sartorius spürt den Einfluss der Pandemie auf mehrere Arten: Die Biotech-Firma trägt durch die Bereitstellung von Forschungs- und Laborequipment zur Entwicklung von Impfstoffen bei und wird als strategisch besonders relevant eingestuft. Die Co-Creation Kultur wurde durch die plötzliche Dezentralisierung der Arbeitsumgebungen vom Unternehmensstandort ins Homeoffice stark provoziert und herausgefordert. 
Die neue Herausforderung war es, die Co-Creation-Kultur des Unternehmens neu zu denken und in kurzer Zeit schnell und effektiv vom Analogen ins Digitale zu überführen. Es galt den Grundsatz Simplifying Progress auch in der neuen Situation anzuwenden und den über 2500 Information Workern innovative Produktentwicklung möglichst einfach zu machen.

\section{The Participation Platform - the digital solution}

Als Antwort auf diese Herausforderung wurden in nur zwei Monaten neue Formate für Tutorials und Webcasts entwickelt und mit den bereits bestehenden Materialien und Inhalten in einem benutzerfreundlichen Co-Creation-Bereich orchestriert. Kontinuierliche Verbesserung, validiertes Lernen und Partizipation: Die Teilnahme am Innovationsprozess auch aus einer remote Situation heraus ist die Kernaufgabe der Participation Platform.

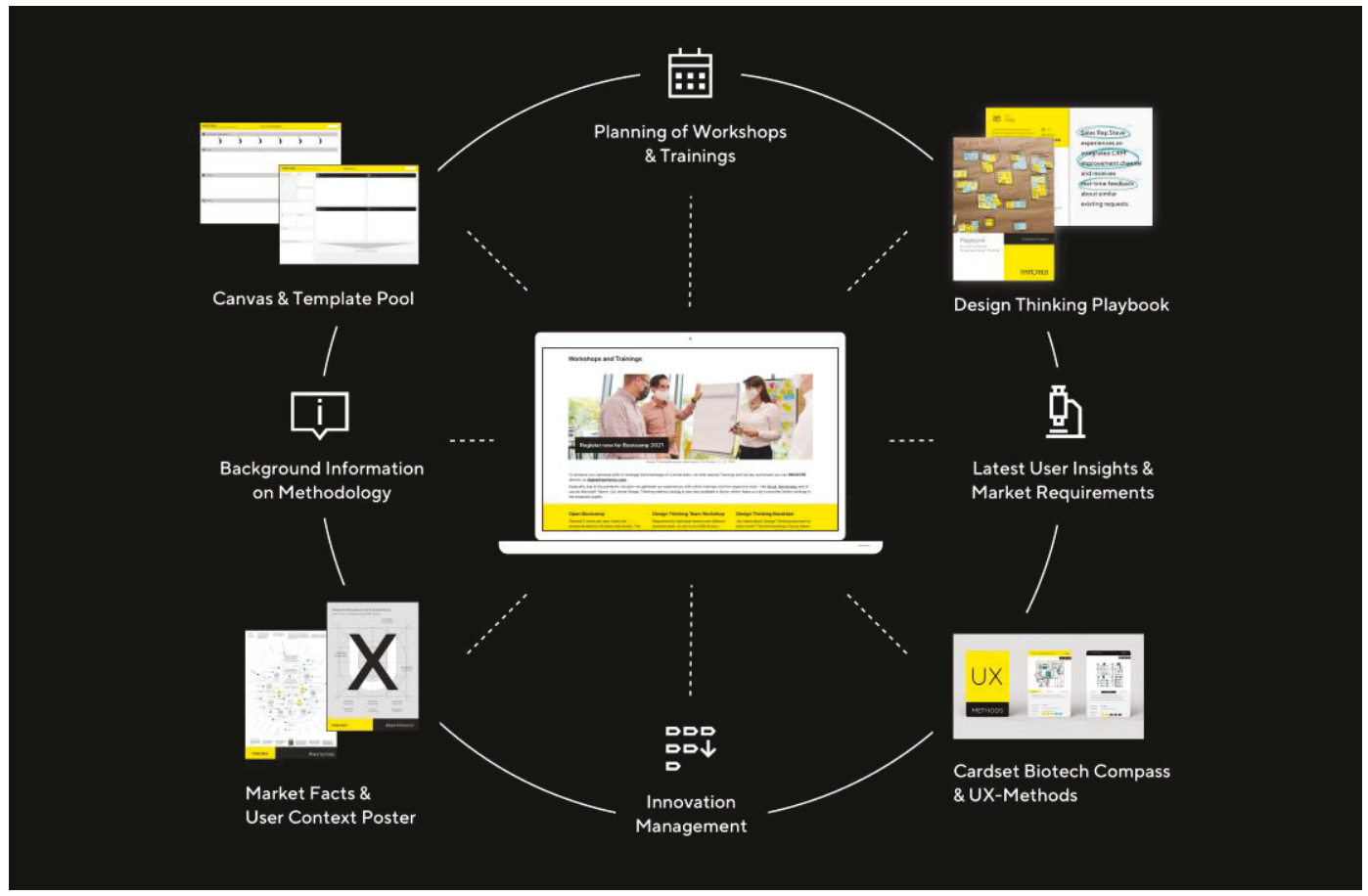

Abbildung 1: Die Participation Platform ermöglicht Zugang zu diversen Inhalten für Co-Creation Workshops 
Über die unternehmensinterne Plattform erhalten Benutzer:innen Zugriff auf Schulungen, aktuelle Nutzer- und Markterkenntnisse, Vorlagen und bewährte Toolkits. Mit den neuesten digitalen Technologien wurde so eine einzigartige Infrastruktur für die Zusammenarbeit geschaffen. Die Plattform wird zur zentralen und schnell zugänglichen Quelle von Informationen und Arbeitsmaterialen, die alle auf den Innovationsprozess und dessen Bedürfnisse im Biotech-Sektor abgestimmt sind (vgl. Abbildung 1).

Folgende Inhalte sind Teil der Plattform:

- Sartorius Design Thinking Method Playbook

- Templates und Methoden-Toolkits

- Digitale Whiteboards für die Zusammenarbeit von Teams in Remote Workshops

- Poster zu Markt- und Nutzerkontexten

- Webcasts für die Mitarbeiter:innen zum selbständigen Eintauchen in die Methoden und Arbeitsweisen

Um den Innovationsprozess herum arrangiert sind:

- Hintergrundinformationen zu Methodologie (zur korrekten Anwendung der Templates und weiteren Workshopmaterialien)

- ein Portal zur Planung von Workshops und Schulungen

Als eines der am schnellsten wachsenden Unternehmen Deutschlands ist der konsequente Ausbau seiner Innovationsinfrastruktur für Sartorius von entscheidender Bedeutung. Das internationale Unternehmen beschäftigt derzeit rund 11000 Mitarbeiter:innen an Standorten auf der ganzen Welt. Durch den Aufbau einer digitalen und standortunabhängigen Co-Creation-Infrastruktur schafft Sartorius eine skalierbare Basis für eine nachhaltige Innovationskultur. Dafür wurden innerhalb weniger Wochen erste Teamleiter:innen geschult, um den ausgewählten Methodensatz anzuwenden und das Design Thinking Method Playbook zu nutzen. Weitere Mitarbeiter:innen werden im Jahr 2021 folgen und basierend auf dem erfolgreichen und durch die ersten Umsetzungsergebnisse bewährten Innovations-Framework fortgebildet.

Im Zuge dieser Veröffentlichung werden drei wesentliche Elemente der neuen digitalen Co-Creation-Kultur im Detail vorgestellt, die zusammengenommen die wichtigsten Innovationstreiber bei Sartorius sind: Das Method-Playbook als methodische Basis für den individuell auf Sartorius abgestimmten Innovationsprozess, der Remote Co-Crea- 
tion Space als unmittelbar verfügbarer digitaler Raum für Workshops und gemeinsames Denken, sowie die Einbindung und Unterstützung der Mitarbeiter:innen von Sartorius, die die Innovationskultur mit Leben füllen.

\section{Method Playbook}

Für die erste Phase des digitalen Rollouts ist bewusst das analoge Method Playbook entwickelt worden, um Akzeptanz zu schaffen und für den kulturellen Wandel zu sensibilisieren. Es wurde im Zuge eines Homeoffice Packages an die Mitarbeiter:innen ausgeliefert, ist aber ebenso als Download auf der Plattform in digitaler Form verfügbar (Abbildung 2).
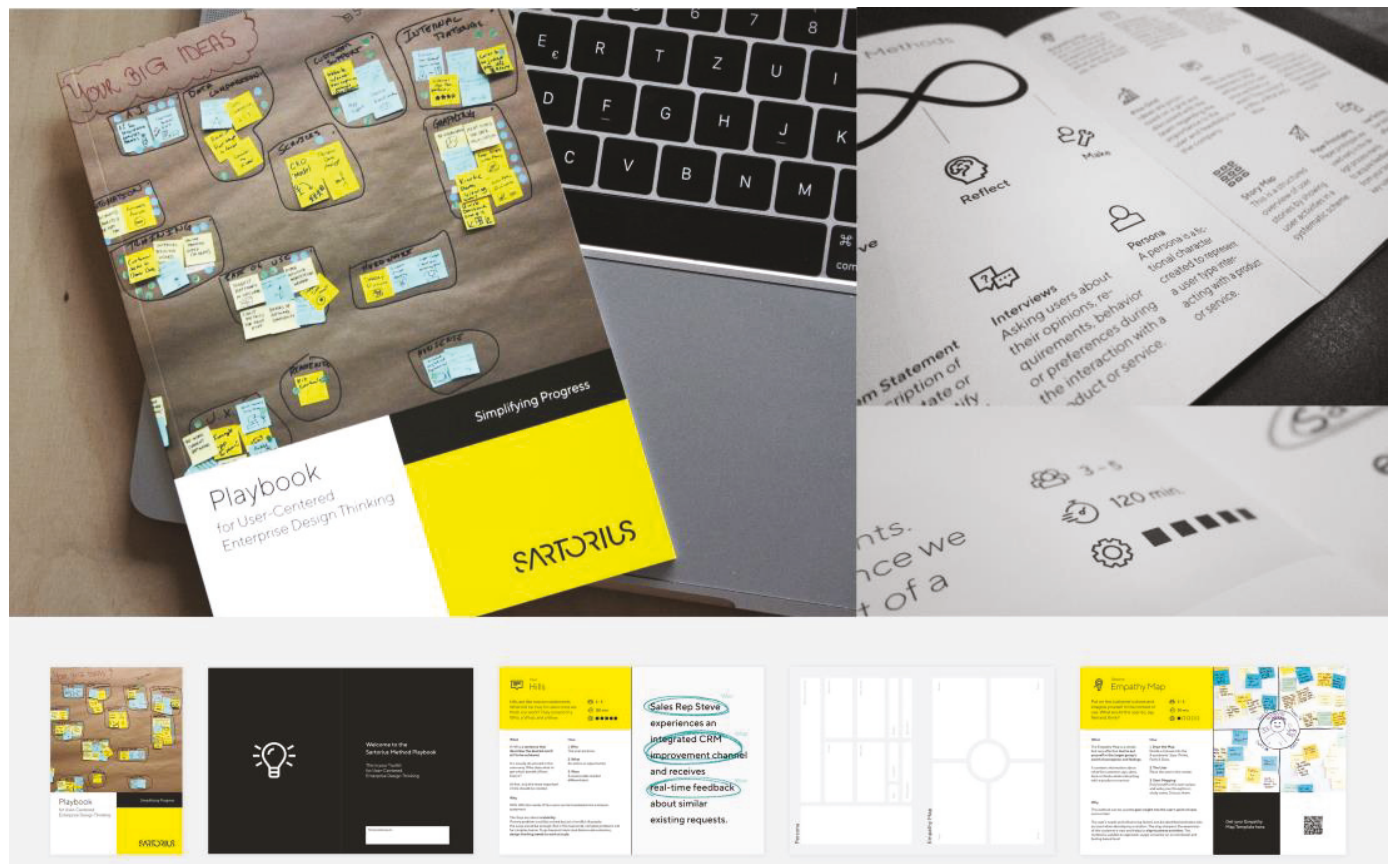

Abbildung 2: Das Method Playbook und einige Beispielseiten

Es umfasst 18 Methoden und Werkzeuge (Abbildung 3), die auf den Innovationsprozess von Sartorius abgestimmt sind. Dies wurde durch das individuelle Layout im neuen Corporate Design unterstrichen. Die Methoden sind didaktisch aufeinander aufbauend im Designprozess vorgestellt, werden erklärt und jeweils mit Beispielen veranschaulicht. Die Methoden sind mit QR-Codes versehen, um eine Verzahnung von 
analogen und digitalen Medien zu erzielen. Dahinter verbergen sich vertiefende Informationen zur Design Thinking Theorie, sowie die digitalen Canvases und Templates zur Onlinenutzung und zum Download und anschließendem Druck (Abbildung 4).

\section{Overview of Methods}
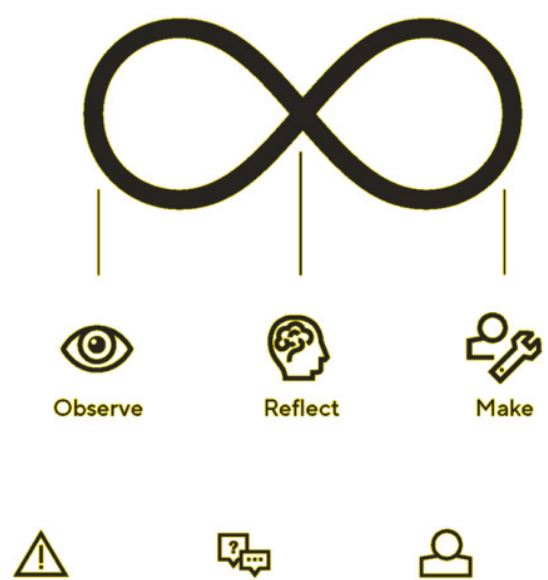

Problem Statement Brief description of the current state or problem to identify the gap to a desired state of a process, service or product.

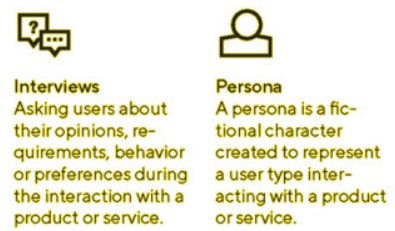

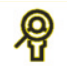

Empathy Map

Put on the shoes of

the customer and

imagine yourself in

the context of use.

What does the user

say, do, feel, think?

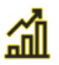

\section{Prio Grid}

Ideas are priori-

tized on a grid and

discussed with the

team regarding the

importance to the

user and feasibility for

the company.

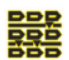

Story Map

This is a structured

overview of user

stories by showing

user activities in a

systematic scheme.

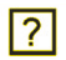

As-Is-Scenario Defining user-specific activities on a scenario map to get a deeper understanding of your persona's workflows and pain points.

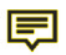

Hills

Hills are like mission statements. What will be true for users once we finish our work? They consist of a Who, a What and a Wow.

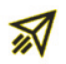

Paper Prototyping Paper prototypes are used early in the design process mainly to acquire feedback from your team and key-users.

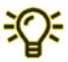

Big Ideas

Brainstormed ideas are sketched on

sticky notes (diverge) Ideas are grouped and voted regarding impact and feasibility (converge).

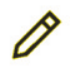

Sketching

Sketching a story-

board is a method to visualize ideas in relation to the context. Draw a sequence of sketches based on the hill.

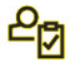

User Testing

User testings are an effective way to test a solution and gather user feedback during all project phases.

Abbildung 3: Eine Übersicht der Methoden, die im Method Playbook von Sartorius aufgeführt und erklärt werden

Die Auswahl der Design Thinking Methoden erfolgte aufgrund der Eignung für digitale und analoge Workshops, die mit Methoden wie der Persona, der Empathy Map oder dem As-Is-Scenario sowohl offline als auch online durchführbar sind.

Damit ist das Method Playbook Startpunkt und Leitfaden für digitale Workshops in interdisziplinären Teams.

\section{Remote Co-Creation Space}

Als digitaler Zwilling fungiert der Remote Co-Creation Space. Hier werden die Workshops durchgeführt und Ideen gesammelt, konzentriert, organisiert, und ausgewählt, Probleme und Herausforderungen erkannt und Lösungen abgeleitet. Es ist eine interaktive 
digitale Workshopumgebung, die eine Zusammenarbeit in interdisziplinären Teams ermöglicht - ortsunabhängig und in Echtzeit. Der Remote Co-Creation Space ist nach dem Whiteboard-Ansatz konzipiert und basiert auf dem Angebot des Unternehmens MIRO. Jede Methode aus dem Method Playbook ist auch hier mit einer eigenen Seite, einem Canvas und zusätzlichen Informationen repräsentiert (vgl. Abbildung 6). So sind die Seiten der Methoden einzeln bespielbar oder in einem Workshop-Setting modular zusammenstellbar. So lässt sich eine Vielzahl von visuellen Kollaborations-Szenarien abbilden.
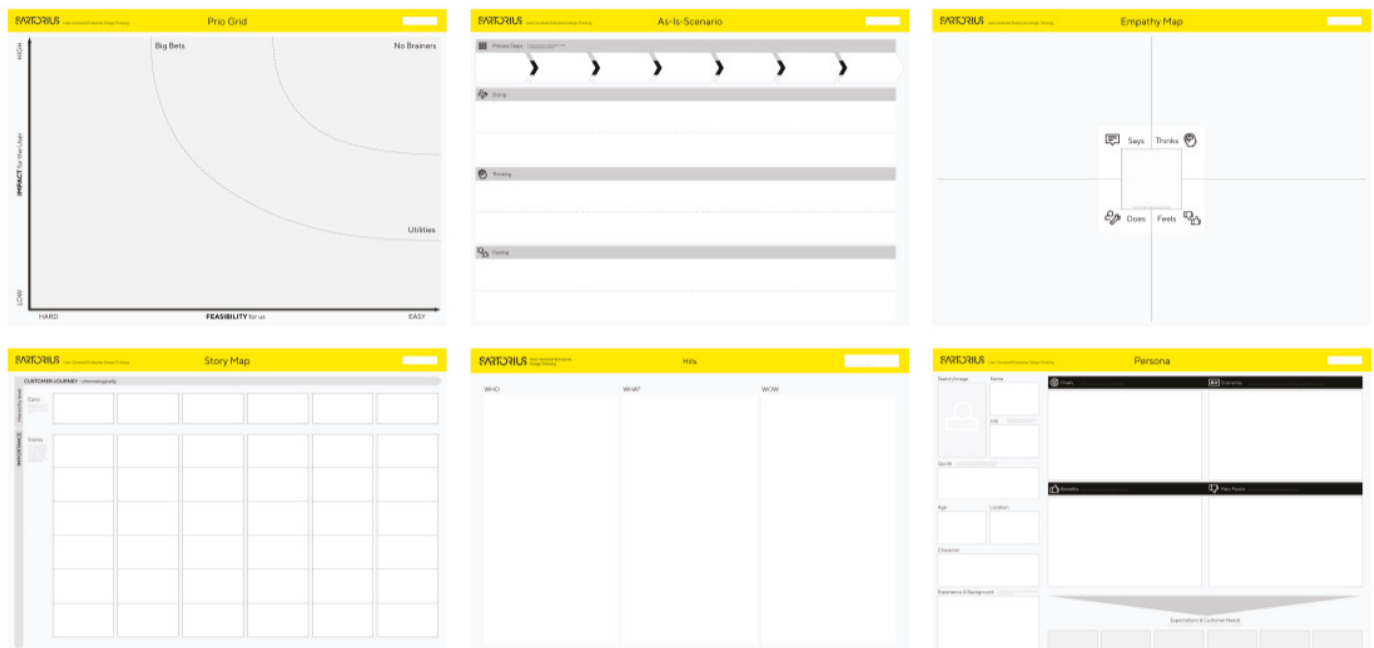

Abbildung 4: Canvas-Vorlagen zum Download und Druck für Vor-Ort-Workshops

Diesem Gedanken folgend gibt es bereits zusammengestellte Workshop-Szenarios in verschiedenen Stufen des Innovationsprozesses. Die passenden Methoden und dazugehörigen Templates aus dem Method Playbook werden vorausgewählt und zu einem Workshop-Template zusammengefasst (vgl. Abbildung 5). Über diese Infrastruktur wurden während der verstärkten Homeoffice Phase im letzten Jahr über 30 Workshops komplett remote abgehalten. Die Moderator:innen dieser Workshops, die teilweise mehrtägig und mit bis zu 20 Teilnehmer:innen durchgeführt wurden, erhielten eine spezielle Einschulung in die effektive Nutzung der Infrastruktur des neuen Remote co-Creation Space. 


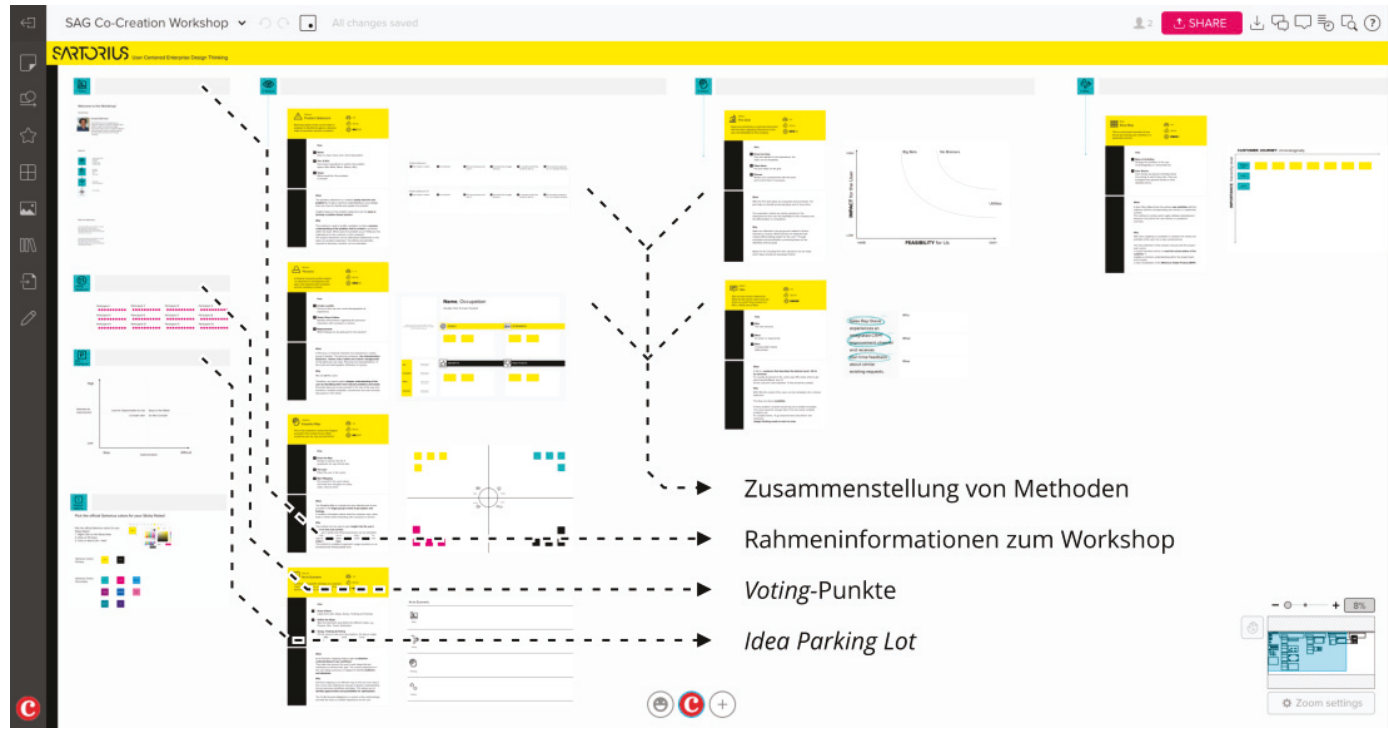

Abbildung 5: Eine Workshop-Vorlage im Corporate Design von Sartorius mit einer Zusammenstellung verschiedener aufeinander aufbauender Methoden

Die browser-basierte digitale Umgebung ist jederzeit zugänglich und vor allem einfach und intuitiv zu bedienen. Auf den virtuellen Whiteboards kann jede:r den Moderator:innen oder den anderen Teammitgliedern folgen und eigene Aktionen live ausführen.

Neben der unkomplizierten Einbindung des gesamten Teams in den aktiven Teil der Veranstaltungen, ist ein weiterer Vorteil dieser digitalen Kollaborationen, dass die Nachbereitung der Inhalte im Vergleich zu Vor-Ort-Workshops vereinfacht wird, weil die Inhalte digital vorliegen und bereits durch die Votingfunktion zusammengefasst und selektiert wurden.

Der Remote Co-Creation Space bietet durch seine Nutzerzentriertheit den optimalen Nährboden für erfolgreiche Kollaboration: Gedankengänge anderer Teilnehmer sind auch remote einfach nachvollziehbar und es entsteht eine partizipative Erfahrung vergleichbar mit einem Event vor Ort.

\section{The human factor - gelebte Innovationskultur durch agile Schulungen und eine Webcast Serie}

Der wichtigste Teil einer erfolgreichen Innovationskultur sind die Mitarbeiter:innen. Methoden alleine sind nicht zielführend - die Schlüssel zum Erfolg sind motivierende 
Kompetenzvermittlung und der einfache Zugriff auf Online-Anleitungen und Vorlagenpools. Die Innovationskultur sollte auch digital funktionieren, die nutzerzentrierte Gestaltung stand deshalb bei der Entwicklung aller Formate im Fokus: Die Verwendung digitaler Co-Creation-Tools reduziert die Vor- \& Nachbereitungszeit, die für Workshops normalerweise anfällt und eliminiert die Kosten für Raummiete, Material, Reisen und Catering. Ein startbereiter Workshopraum ist jederzeit nur einen Klick entfernt.

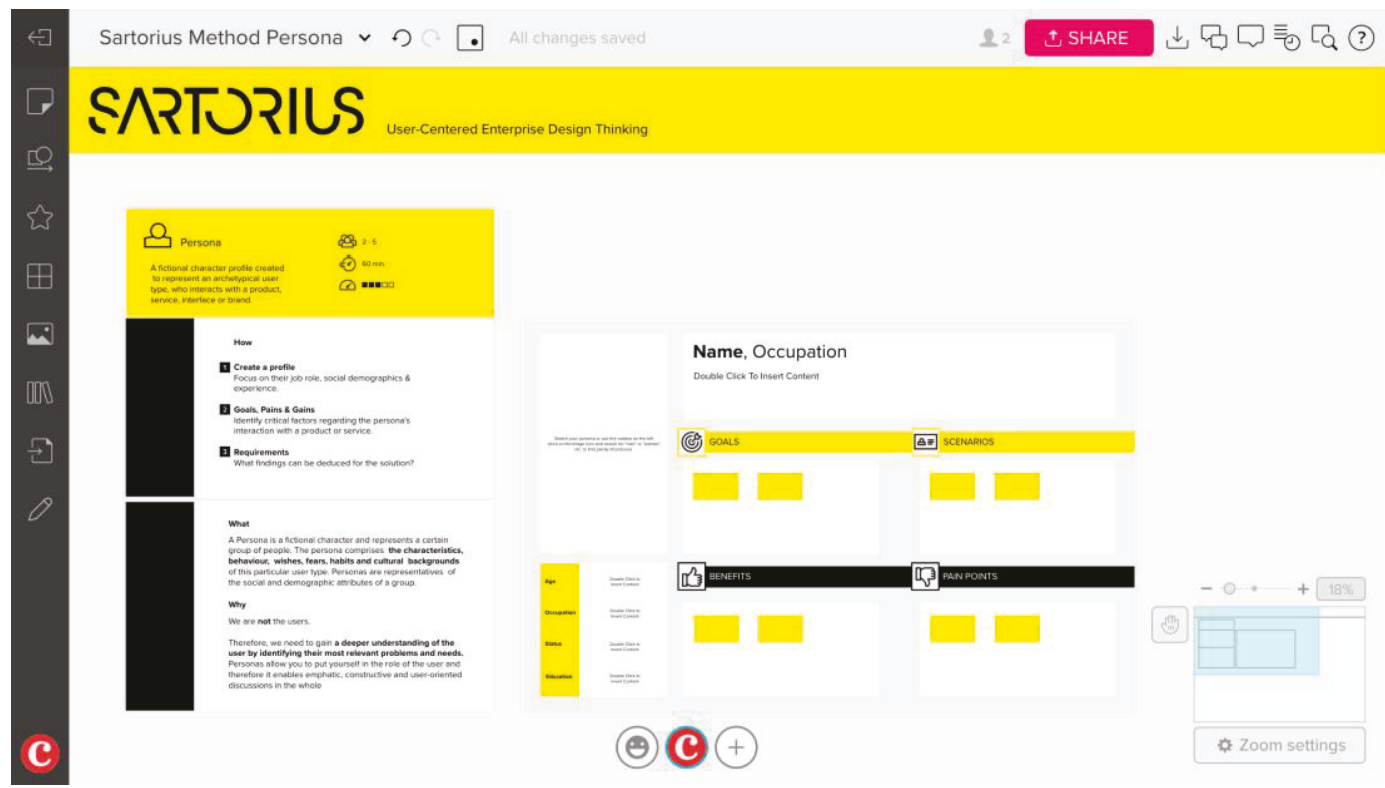

Abbildung 6: Die Design Thinking Methode Persona auf einem digitalen Whiteboard

Insbesondere wurde im Zuge des Rollouts der Plattform ein Train-the-Trainer-Programm initiiert. Durch die Schulung von weiteren Mitarbeiter:innen mithilfe eines Slidedecks zu den agilen Methoden aus dem Method Playbook werden die Kompetenzen für die digitale Innovation zügig und effektiv in das Unternehmen eingebracht.

Just-in-time-Unterstützung bietet die entwickelte Webcast-Serie. Dezentral und on-demand können weitere Hintergrundinformationen, Tipps und Tricks und Best Practice Beispiele zu den Plattforminhalten in drei bis fünfminütigen Videos von allen Mitarbeiter:innen abgerufen werden. Dadurch wird die Skalierung der neuen remote Arbeits- 
weisen unterstützt. Jederzeit abrufbar und ohne zusätzlichen Support weiterer Trainer:innen finden die Mitarbeiter:innen selbständig in die Themen und entscheiden individuell, was für sie im jeweiligen Kontext relevant ist.

\section{Fazit}

Jedes Format und jeder Berührungspunkt wurde nach einem partizipativen Ansatz entworfen und validiert, um die co-kreative Arbeit und das innovative Denken von allen Teilnehmer:innen zu fördern. Die Benutzer:innen finden eine Umgebung, die einen schnellen Zugriff auf Methoden, Tools und Erkenntnisse bietet und so neue Anreize zur Entwicklung neuer Ideen schafft. Innovative digitale Trainingsformate, die von Expert:innen geleitet werden, schaffen eine Startup-ähnliche Atmosphäre.

Die digitale Plattform für Innovation bietet einige Vorteile für Sartorius:

- Zeiteinsparung (An- und Abreise, Vor- und Nachbereitung der Workshop-Sessions)

- Ressourceneinsparung (Räume, Materialien, Reisekosten, etc.)

- Erhöhung der Akzeptanz der Innovationsmethoden in den Teams und spürbare Steigerung der aktiven Partizipation

- Zugang zu Methoden, Informationen und Werkzeugen

Mit seinen Methoden und digitalen Formaten, die individuell auf das Unternehmen zugeschnitten sind, geht Sartorius neue Wege und prägt die Innovationskultur des Unternehmens nachhaltig. Jüngste Erfolge im Bereich der Produktentwicklung und die Einführung neuer Serviceangebote beweisen, dass der Remote Innovation Ansatz bereits Früchte trägt und langfristig effektiv funktioniert.

\section{Kontakt}

Dipl.-Des. Oliver Gerstheimer, Managing Partner

Philipp Schütz, M. A., Senior UX-Designer

Philipp Englisch, M. Sc., UX-Designer

chilli mind $\mathrm{GmbH}$

Königstor 2334117 Kassel

www.chilli-mind.com
Erhard Wimmer

User and Customer Experience Design

Business Process Management

Sartorius Stedim Biotech GmbH

August-Spindler Straße 11, 37079 Göttingen

www.sartorius.com 